\title{
A tool for computation of electrical losses in wind farms grids
}

\author{
L. Rouco \\ Universidad Pontificia Comillas \\ Alberto Aguilera, 23, 28015 Madrid, Spain \\ I. Campos, M. Hernández, J. C. Pérez Campión and I. Gómez de Olea \\ Iberdrola \\ Tomás Redondo 1, 28033 Madrid, Spain
}

\begin{abstract}
Accurate calculation of electrical losses of wind farm grids is needed in the economic evaluation of wind generation projects. This paper describes a tool for computation of electrical losses in wind farm grids. It makes use of the probability density function (PDF) of the wind speed of each wind generator to develop a set of generation scenarios.
\end{abstract}

Keywords. Wind generation, Weibull probability density function, electrical losses.

\section{Introduction}

Depending on the grid connection approach, several schemes of wind farm development can be found. One scheme consists of connecting single wind generators to the distribution grid. It is aimed at a distributed integration of wind generation. Such scheme is very common in countries like Denmark and Germany. Other approach consists of connecting several wind farms (wind farm development) to either subtransmission or transmission grids. Such scheme is frequently used in countries like Spain and the USA since it facilitates the large scale integration of wind generation.

Under the later scheme, wind farms need a complex grid to transmit wind generator production to the point of common coupling. The components of such grid are wind generator step-up transformer, medium voltage grid that connect wind generators to wind farm substation, medium voltage-high voltage wind farm transformer and high voltage grid up to the point of common coupling.

Accurate calculation of electrical losses is needed for economic evaluation of each project. Such calculation cannot be done in just a single scenario (i.e. $80 \%$ of wind farm nameplate installed capacity). This paper proposes to consider the probability density function (PDF) of the wind speed of each wind generator to develop a set of generation scenarios. PDFs of the wind speed of each wind generator are available from the wind farm production study.

The paper describes a tool to compute electric losses in the overall electric infrastructure of wind farms. The tool uses the power flow data model and the solution algorithms of commercially available program package [2]. An actual example is used to illustrate the tool.

The paper is organized as follows. Section 2 explains the scenario development. Section 3 provides an overview of the tool. Section 4 describes the test case proposed to illustrate the tool. Section 5 contains illustrative results. Section 6 provides the conclusions of the paper.

\section{Scenario development}

Scenario development consists of determining a set of generation snapshots of equal duration. Scenarios are determined from the PDF of the wind speed of each wind generator of the wind farms and the wind speed-power characteristic of each wind generator.

Wind speed probability is usually described by a Weibull PDF [1]:

$$
f(x)=\frac{\alpha}{\beta^{\alpha}} x^{\alpha-1} e^{-\left(\frac{x}{\beta}\right)^{\alpha}}
$$

where $x$ is the random variable (wind speed) and $\alpha$ and $\beta$ are parameters that determine the shape of the PDF.

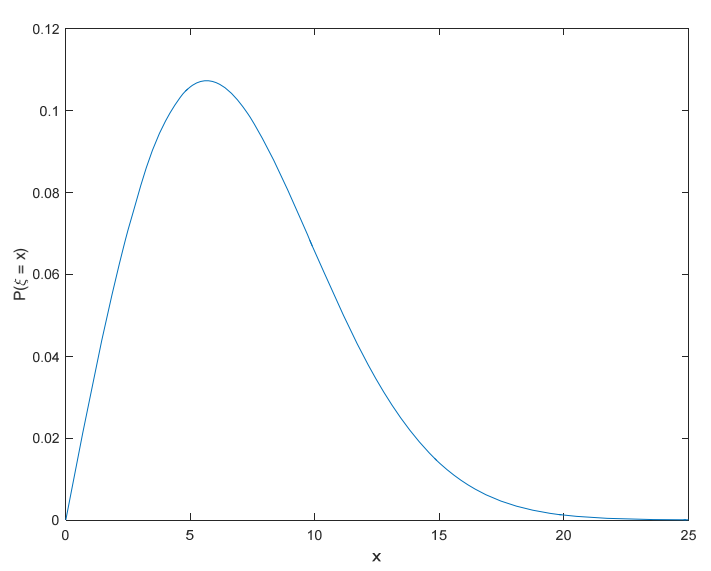

Figure 1: Weibull PDF. 
Figure 1 shows the Weibull PDFn with $\alpha=2$ and $\beta=6$. The cumulative probability function (CPF) corresponding to a Weibull PDF becomes:

$$
F(x)=1-e^{-\left(\frac{x}{\beta}\right)^{\alpha}}
$$

Figure 2 shows the CPF corresponding to a Weibull PDF with $\alpha=2$ and $\beta=6$. The complementary of the CPF corresponding to a Weibull PDF results in:

$$
F^{\prime}(x)=1-F(x)=e^{-\left(\frac{x}{\beta}\right)^{\alpha}}
$$

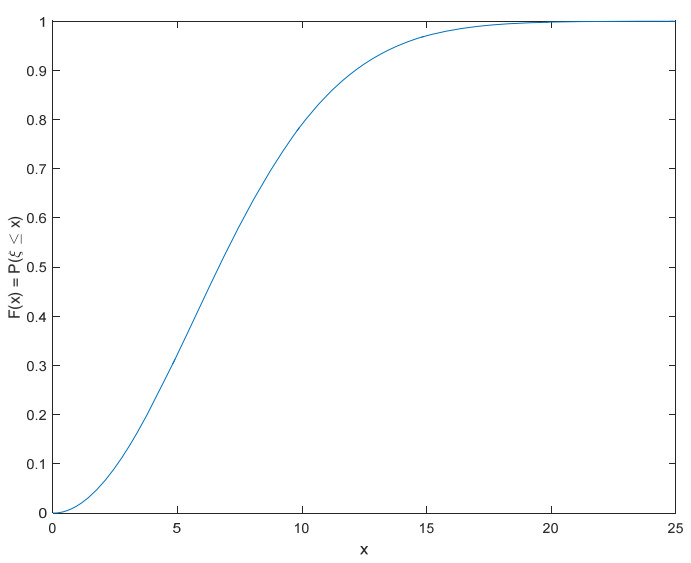

Figure 2: CPF of a Weibull PDF.

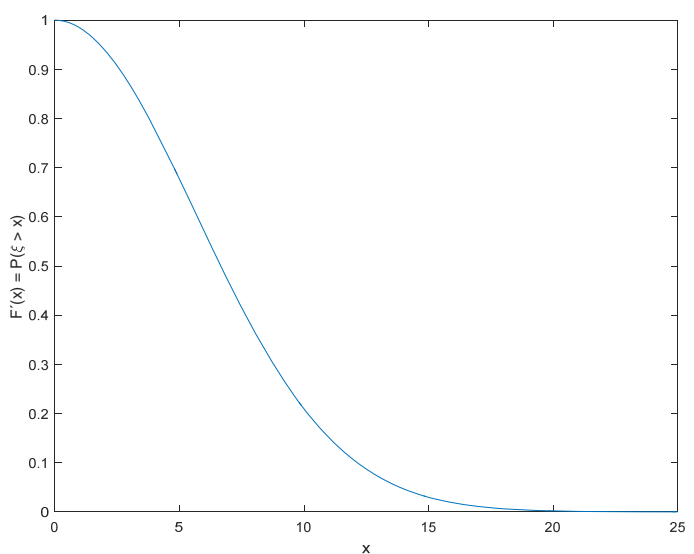

Figure 3: Complementary of the CPF corresponding to a Weibull PDF.

Figure 3 shows the complementary of the CPF corresponding to a Weibull PDF with $\alpha=2$ and $\beta=6$. Power generation dispatch studies make use of the socalled generation-duration curve [3] which is the complementary of the cumulative probability function expressing the probability as the duration in hours of a year. Hence, the wind speed-duration curve can be formulated as:

$$
d=8760 \cdot e^{-\left(\frac{w}{\beta}\right)^{\alpha}}
$$

where $w$ is the wind speed and $d$ is the duration (number of hours in which wind speed is over $w$ ).

Figure 4 shows the wind speed-duration curve corresponding to a Weibull PDF with $\alpha=2$ and $\beta=6$. Building wind farm generating scenarios with wind generators that exhibit different Weibull PDF requires to consider scenarios of identical duration. The wind speed $w$ that corresponds to a duration $d$ is given by:

$$
w=\beta\left[-\log \left(\frac{d}{8760}\right)\right]^{\frac{1}{\alpha}}
$$

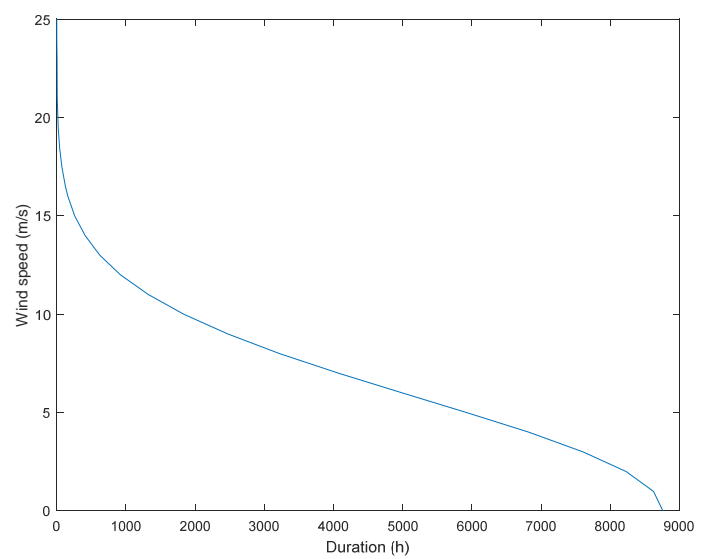

Figure 4: Wind speed-duration curve corresponding to a Weibull PDF.

The continuous wind speed-duration curve is discretized using the trapezoidal rule according to

$$
\bar{w}_{i}=\frac{w_{i-1}+w_{i}}{2}=\frac{\beta\left[-\log \left(\frac{i-1}{N_{s}}\right)\right]^{\frac{1}{\alpha}}+\beta\left[-\log \left(\frac{i}{N_{s}}\right)\right]^{\frac{1}{\alpha}}}{2}
$$

where $N_{s}$ is the number of scenarios. Generation supplied by a wind generator is obtained from the discretized wind speed duration curve and power characteristic of the wind generator.

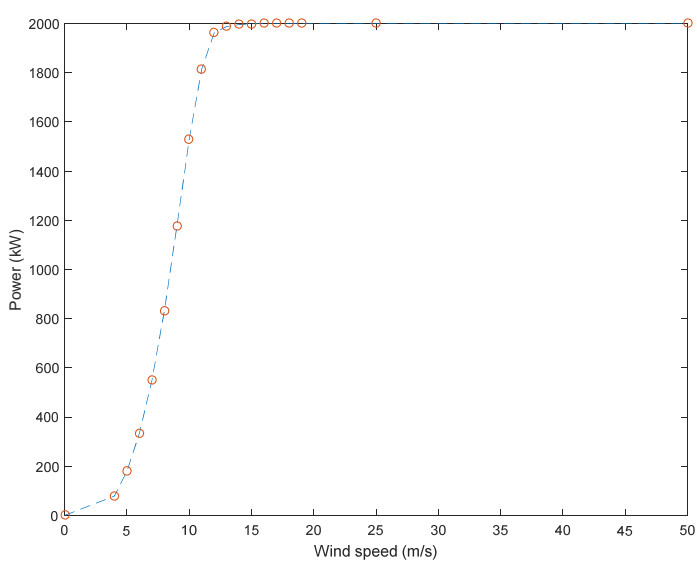

Figure 5: Power curve of Gamesa G87 wind generator. 
Figure 5 displays the power curve of a Gamesa G87 wind generator [4]. As the power curve of a wind generator is defined by a set of points, the active power generation corresponding to the $\mathrm{i}$-th scenario is determined by linear interpolation of two points of the power curve of the wind generator:

$$
\begin{aligned}
& \bar{P}_{i}=P_{k}+\frac{P_{k+1}-P_{k}}{w_{k+1}-w_{k}}\left(\bar{w}_{i}-w_{k}\right) \\
& w_{k}<\bar{w}_{i} \leq w_{k+1}
\end{aligned}
$$

Figure 6 depicts the scenario bar diagram of a Gamesa G87 wind generator when 20 scenarios have been considered.

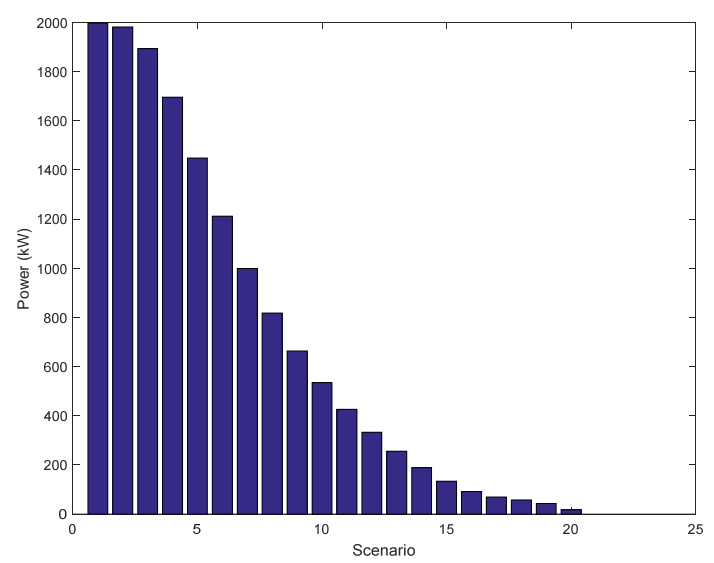

Figure 6: Power scenarios of a Gamesa G87 wind generator.

\section{Tool overview}

The tool is based on the power flow data model and the power solution algorithms of a commercially available simulation package (Siemens PTI PSS/E, [2]). A Microsoft Access data base is used to handle input data and to produce a set of auxiliary files. PSS/E power flow raw data file is among those auxiliary files. Input data are provided in two excel files: 'Data.xls' and 'Catalogue.xls'. Output results are provided in an excel file 'Results.xls'. Figure 7 provides an overview of the tool.

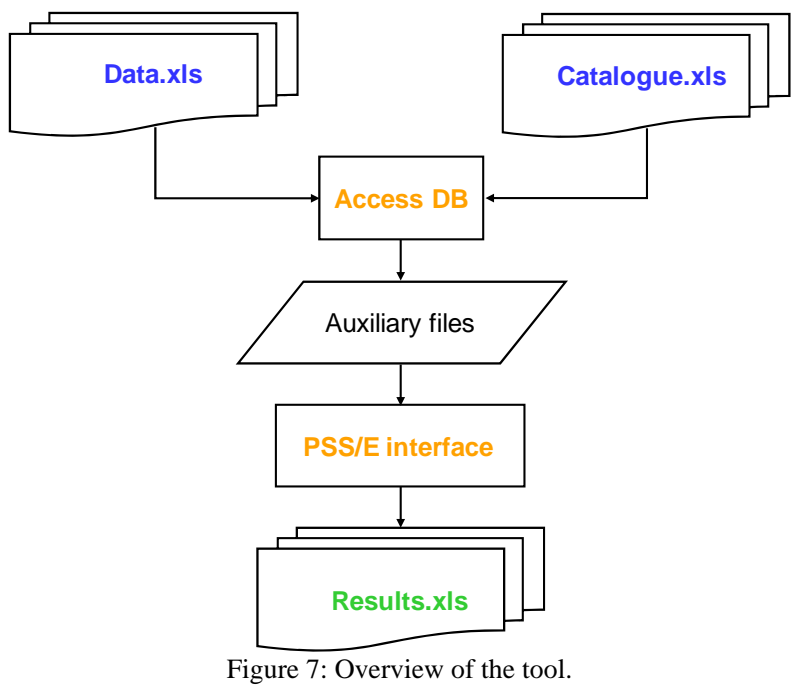

The excel book 'Data.xls' provides the specific data of an installation. It contains the following sheets:

- External grid

- HV buses

- $\mathrm{HV} / \mathrm{HV}$ two winding transformers

- $\mathrm{HV} / \mathrm{HV}$ three winding transformers

- HV transmission lines

- HV/MV Transformers

- Shunt devices

- Switching stations

- MV distribution lines

- Wind generators

- Fixed injections

The excel book 'Catalogue.xls' provides parameters of installation components like HV transmission lines and MV distribution lines and wind generators. The catalogue facilitates building the 'data.xls' excel book. The catalogue contains the following sheets:

- HV transmission lines

- MV distribution lines

- Wind generators

Three losses studies can be alternatively undertaken:

- Hourly study

- Yearly study

- Sensitivity study

The hourly study just simulates a scenario defined by a fraction of the nameplate installed capacity of each wind generator. The yearly study simulates the operation of the wind farm development over a year. The number of scenarios can be selected by the user.

The excel book 'Results.xls' contains the following sheets:

- Power report

- Energy report

Table I: Nameplate installed capacity of Fuentes de la Alcarria installation.

\begin{tabular}{|l|r|}
\hline Wind farm & Installed capacity (MW) \\
\hline CLARES & 32 \\
EL_CABEZUELO & 30 \\
ESCALON & 30 \\
LUZON_NORTE & 38 \\
MARANCHON_I & 18 \\
MARANCHON_IV & 48 \\
MARANCHON_SU & 12 \\
SIERRA_MENER & 40 \\
OTROS_PROM & 150 \\
PROMOTOR_A & 25 \\
PROMOTOR_B & 35 \\
PROMOTOR_C & 40 \\
\hline TOTAL & 498 \\
\hline
\end{tabular}

\section{Test case}

The tool will be illustrated with Fuentes de la Alcarria wind generation development. Fuentes de la Alcarria wind generation development comprises of 12 wind 
farms connected through a common infrastructure to the Fuentes de la Alcarria $400 \mathrm{kV}$ substation. Table I details the nameplate installed capacity of the wind farms of Fuentes de la Alcarria which amounts almost 500 MW. Iberdrola Renovables owns and operates wind farms which nameplate installed capacity is 248 MW. Figure 8 shows the single line diagram of Fuentes de la Alcarria wind generation development. Wind farms are connected to Fuentes de la Alcarria $400 \mathrm{kV}$ substation through a 132 $\mathrm{kV}$ subtransmission grid and a 450 MVA, $400 \mathrm{kV} / 132$ $\mathrm{kV}$ transformer.

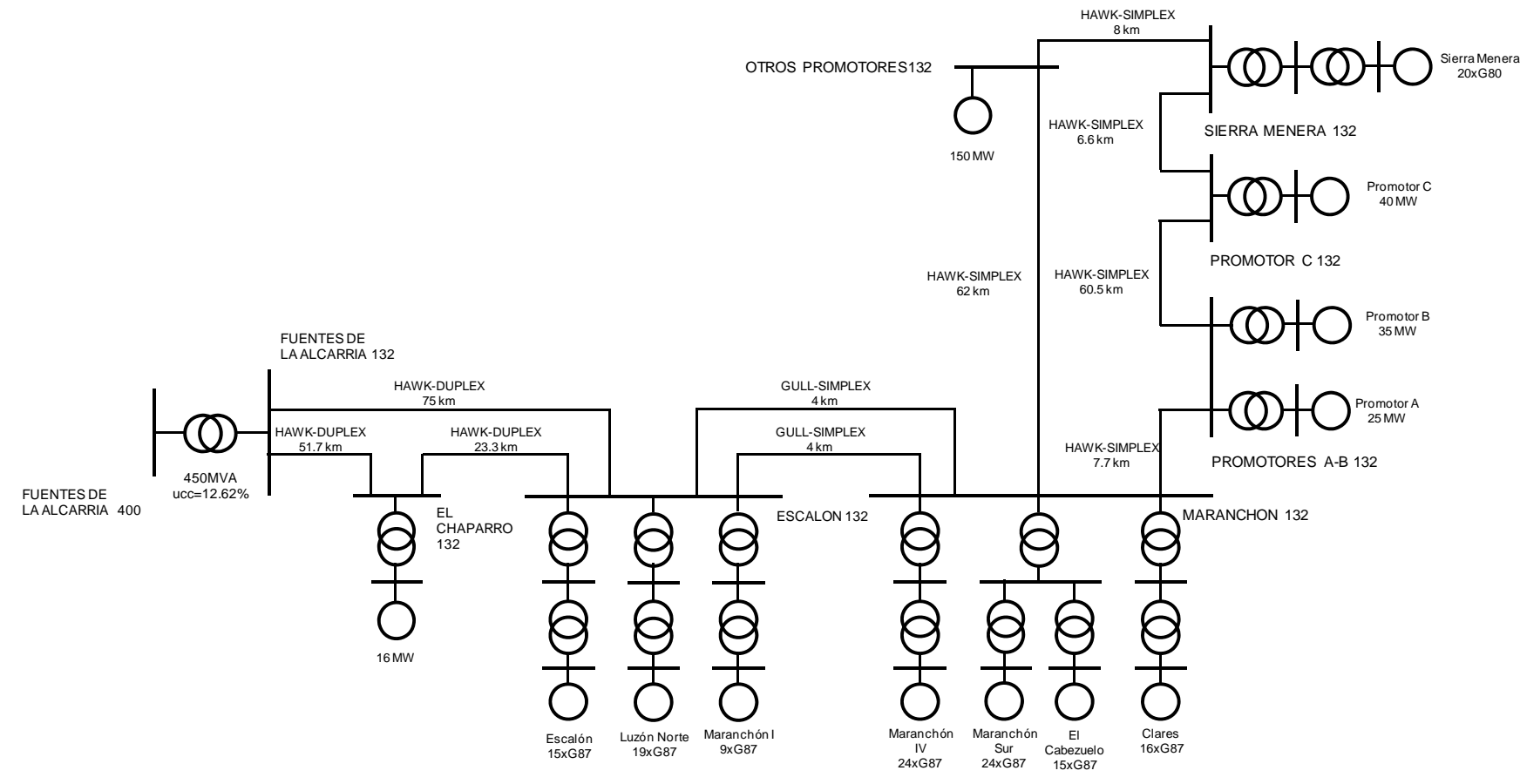

Note: Wind farm transformers: $55 \mathrm{MVA}, 132 \mathrm{kV} / 20 \mathrm{kV}$, ucc $=10 \%$

Figure 8: Single-line diagram of test case.

Table II: Comparison of Weibull PDF based scenarios with a single $80 \%$ nameplate generation scenario.

\begin{tabular}{|c|c|c|c|c|c|c|c|c|}
\hline & \multicolumn{3}{|c|}{ Wind generator output: Weibull PDF } & \multicolumn{5}{|c|}{ Wind generator output: $80 \%$ nameplate installed capacity } \\
\hline & Generation (MWh) & Losses (MWh) & Losses (\%) & Power (MW) & Losses (MW) & Generation (MWh) & Losses (MWh) & Losses (\%) \\
\hline CLARES & 88462.86 & 1334.9 & 1.51 & 25.6 & 0.44 & 224256 & 3854.4 & 1.72 \\
\hline EL_CABEZUELO & 76842.72 & 654.24 & 0.85 & 24 & 0.24 & 210240 & 2102.4 & 1 \\
\hline ESCALON & 82129.38 & 806.26 & 0.98 & 24 & 0.28 & 210240 & 2452.8 & 1.17 \\
\hline LUZON_NORTE & 92339.16 & 1162.18 & 1.26 & 30.4 & 0.47 & 266304 & 4117.2 & 1.55 \\
\hline MARANCHON_I & 50904.36 & 727.08 & 1.43 & 14.4 & 0.25 & 126144 & 2190 & 1.74 \\
\hline MARANCHON_IV & 114668.4 & 3375.93 & 2.94 & 38.4 & 1.29 & 336384 & 11300.4 & 3.36 \\
\hline MARANCHON_SU & 30046.8 & 359.37 & 1.2 & 9.6 & 0.13 & 84096 & 1138.8 & 1.35 \\
\hline SIERRA_MENER & 108015.18 & 1156.66 & 1.07 & 32 & 0.4 & 280320 & 3504 & 1.25 \\
\hline INSTALLATION & 0 & 13320.65 & & 0 & 4.77 & 0 & 41785.2 & \\
\hline TOTAL & 643408.86 & $22897.27^{\prime}$ & 3.56 & 198.4 & 8.27 & 1737984 & 72445.2 & 4.17 \\
\hline
\end{tabular}

\section{Simulation results}

\subsection{Constant generation versus generation according to Weibull PDF}

The value of developing wind generation scenarios from Weibull PDF is discussed firstly. Table II compares Weibull PDF based scenarios with a single $80 \%$ nameplate generation scenario. If the $80 \%$ nameplate would be extended to the yearly generation and losses would have become 1737984 MWh and 72445 MWh respectively. In contrast, when Weibull PDF based scenarios are assumed, the yearly generation and losses would have become 642408 MWh and 22898 MWh. In the later case the losses are $3.56 \%$ of the total generation whereas in the former case the losses are $4.17 \%$. It should be noted the $80 \%$ nameplate generation scenario approach overestimates the electrical losses.

\subsection{Detailed versus aggregated wind farm models}

The tool can handle either detailed models or aggregated models of wind farms. Aggregated models neglect the wind farm MV distribution grids and represent all wind generators delivering their output though a $\mathrm{HV} / \mathrm{MV}$ transformer as a single one. The aggregated wind farm model assumes a single set of parameters of the Weibull PDF. Aggregated models are of interest in case loss factor calculations and optimal flow solutions.

Table III compares generation and losses of the detailed and aggregated models. The detailed model results in higher losses as the wind farm MV distribution grid has not been represented. In contrast, due to the fact a single set of parameters of the Weibull PDF is assumed by the aggregated model, it overestimates the generation. 
Table III: Comparison detailed and aggregated models.

\begin{tabular}{|l|r|r|}
\cline { 2 - 3 } \multicolumn{1}{c|}{} & \multicolumn{1}{c|}{ Detailed model } & Aggregated model \\
\hline Generation (MWh) & 643408.86 & 645515.64 \\
Losses (MWh) & 22897.25 & 17353.36 \\
Losses (\%) & 3.56 & 2.69 \\
\hline
\end{tabular}

Figure 9 and Figure 10 compares generation and losses, respectively, with detailed and aggregated models of wind farms along the scenarios considered.

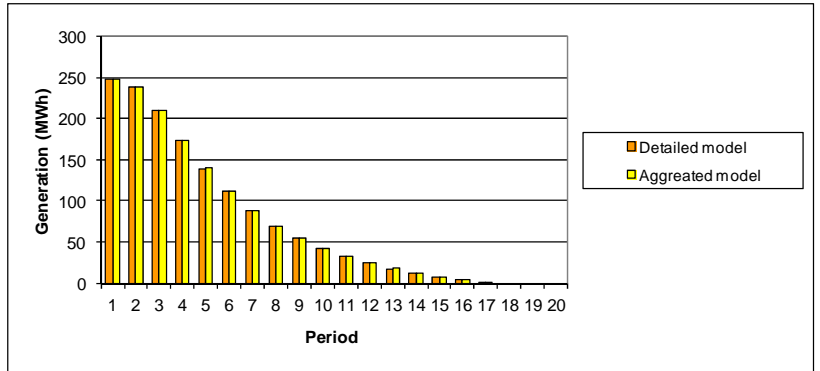

Figure 9: Comparison of generation with detailed and aggregated models.

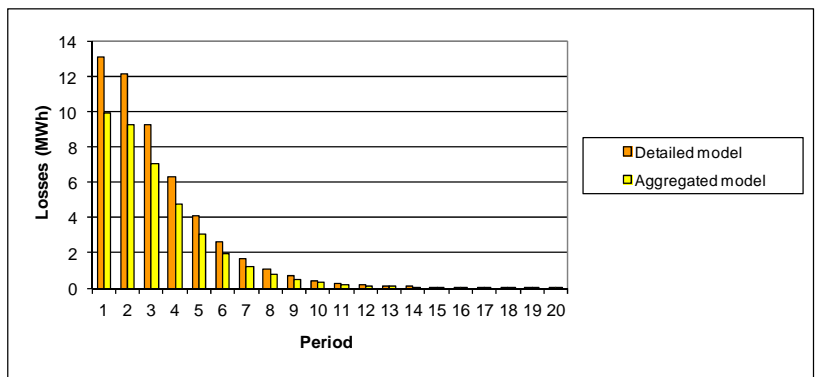

Figure 10: Comparison of losses with detailed and aggregated models.

Table IV compares the losses in each of the wind farms which Weibull PDF is available assuming detailed and aggregated models. It is interesting to note that the losses of wind farm represented by detailed models are not uniform whereas the losses of wind farms represented by aggregated models are. It suggests different design criteria of MV distribution grids of wind farm.

Table IV: Comparison detailed and aggregated models.

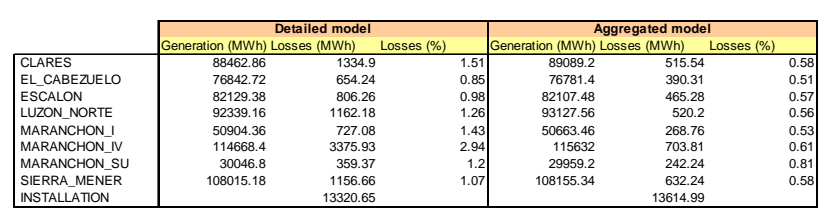

\subsection{Losses sensitivity}

The tool can also perform sensitivity studies like the sensitivity of losses with respect to active power generation and reactive power generation. Figure 11 shows the losses sensivity with respect to wind generation (active powe). A typical quadratic curve has been obtained as expected. Figure 12 depicts the losses sensitivity with respect to reactive power generation.

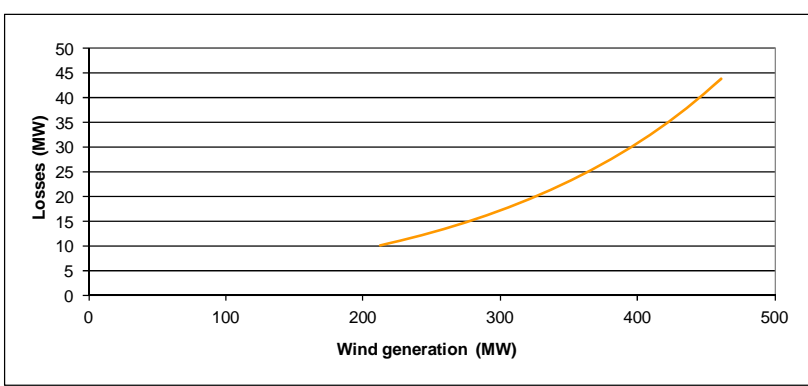

Figure 11: Losses sensitivity with respect o wind generation.

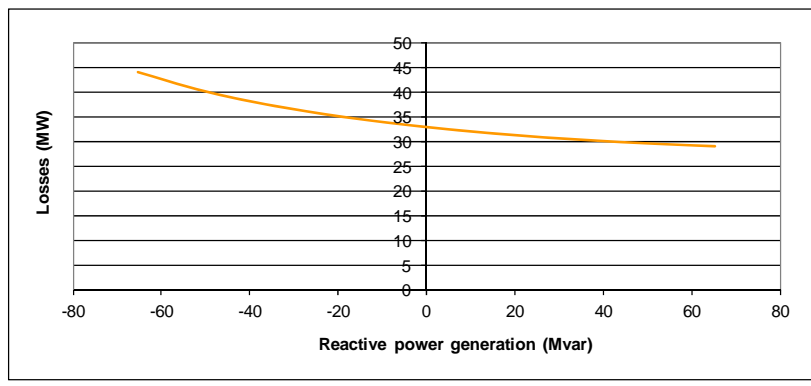

Figure 12: Losses sensitivity with respect to reactive power generation.

\section{Conclusions}

This paper has describeb a tool for computation of electrical losses in wind farm grids. It makes use of the Weibull PDF of the wind speed of each wind generator to develop a set of generation scenarios.

The tool is based on the power flow data model and the power solution algorithms of a commercially available simulation package (Siemens PTI PSS/E). A Microsoft Access data base is used to handle input data and to produce a set of auxiliary files.

The tool has been illustrated with Fuentes de la Alcarria wind generation development. Fuentes de la Alcarria wind generation development comprises of 12 wind farms connected through a common infrastructure to the Fuentes de la Alcarria $400 \mathrm{kV}$ substation.

\section{References}

[1] G. L. Johnson, "Economic Design of Wind Electric Systems", IEEE Transactions on Power Apparatus and Systems, Vol. PAS97, No. 2, February 1978, pp. $554-562$.

[2] Siemens PTI, "Power System Simulator for Engineering PSS/E", http://w3.siemens.com/smartgrid/global/en/products-systemssolutions/software-solutions/planning-data-managementsoftware/planning-simulation/pages/pss-e.aspx.

[3] A.J. Wood, B. F. Wollenber, "Power Generation, Operation and Control”, John Wiley and Sons, 1984.

[4] Gamesa, G87-2MW, http://www.wind-powerprogram.com/Library/Turbine\%20leaflets/Gamesa/Gamesa\%20G 87\%202mw.pdf

\section{Acknowledgements}

The tool was coded and tested by R. Casanova and M.I. Navarrete. I. Azpiri and R. Veguillas from Iberdrola Renovables contributed to the initial stages of its development. 